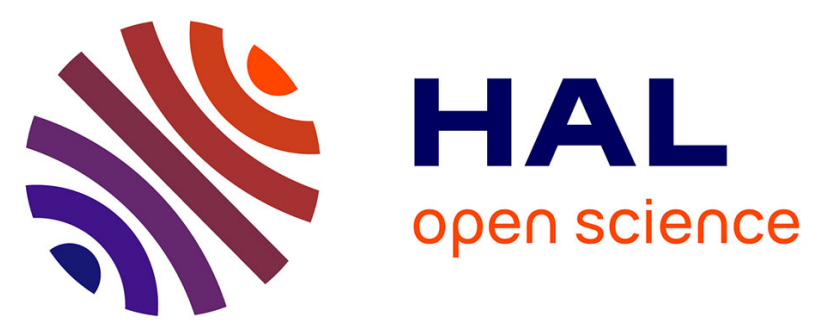

\title{
Management of initial orthostatic hypotension: lower body muscle tensing attenuates the transient arterial blood pressure decrease upon standing from squatting
}

C. T. Paul Krediet, Ingeborg K. Go-Schön, Yu-Sok Kim, Mark Linzer, Johannes J. van Lieshout, Wouter Wieling, C. T. Paul Krediet

\section{- To cite this version:}

C. T. Paul Krediet, Ingeborg K. Go-Schön, Yu-Sok Kim, Mark Linzer, Johannes J. van Lieshout, et al.. Management of initial orthostatic hypotension: lower body muscle tensing attenuates the transient arterial blood pressure decrease upon standing from squatting. Clinical Science, 2007, 113 (10), pp.401-407. 10.1042/CS20070064 . hal-00479369

\section{HAL Id: hal-00479369 \\ https://hal.science/hal-00479369}

Submitted on 30 Apr 2010

HAL is a multi-disciplinary open access archive for the deposit and dissemination of scientific research documents, whether they are published or not. The documents may come from teaching and research institutions in France or abroad, or from public or private research centers.
L'archive ouverte pluridisciplinaire HAL, est destinée au dépôt et à la diffusion de documents scientifiques de niveau recherche, publiés ou non, émanant des établissements d'enseignement et de recherche français ou étrangers, des laboratoires publics ou privés. 
MANUSCRIPT CS2007/0064

Management of initial orthostatic hypotension: Lower body muscle tensing attenuates the transient arterial blood pressure decrease upon standing from squatting

C. T. Paul Krediet MD ${ }^{1}$, Ingeborg K. Go-Schön ${ }^{1}$, Yu-Sok Kim MD ${ }^{1}$, Mark Linzer MD ${ }^{2}$, Johannes J. van Lieshout MD PhD ${ }^{1}$ and Wouter Wieling MD PhD ${ }^{1}$

\footnotetext{
Affiliations: ${ }^{1}$ Department of Internal Medicine, Academic Medical Center at the University of Amsterdam, Meibergdreef 9, 1105AZ, Amsterdam, The Netherlands

${ }^{2}$ Department of Medicine, University of Wisconsin, 2828 Marshall Court, Madison, Wisconsin 53705, USA
}

Brief title: Management of initial orthostatic hypotension

Key words: Orthostatic hypotension, Posture, Pre-syncope, Syncope, Therapy

Word count: 2670 (excl. refs)

Address for correspondence:

CTP Krediet, University of Amsterdam, Academic Medical Center, Department of Internal Medicine, F4-222, Meibergdreef 9, 1105AZ, Amsterdam, The Netherlands. Tel: *31-20-5669111; Fax: *31-206919658; E-mail: C.T.Krediet@amc.uva.nl 


\begin{abstract}
Initial orthostatic hypotension ( $\mathrm{IOH}$ ) comprises symptoms of cerebral hypoperfusion caused by an abnormally large transient mean arterial blood pressure (MAP) decrease 5-15 s after arising from a supine, sitting or squatting position. Few treatment options are available. We set out to test the hypothesis that lower body muscle tensing (LBMT) attenuates IOH after rising from squatting and its symptoms in daily life. Thirteen IOH patients ( 9 males, 27 years) rose twice from squatting; once with LBMT. In addition seven healthy volunteers ( 5 males, median age 27 years) were studied in a crossover study design. They stood up from the squatting position three times, once combined with LBMT. Blood pressure (Finometer) was continuously measured and cardiac output (CO by Modelflow) and total peripheral resistance (TPR) were computed. MAP, CO and TPR were compared with and without LBMT. Using a questionnaire, the perceived effectiveness of LBMT in the patients' daily lives was evaluated. With LBMT the minimal MAP after standing up was higher, in both groups $(19 \mathrm{mmHg}$ in patients and $13 \mathrm{mmHg}$ in healthy subjects). In healthy subjects the underlying mechanism was a blunted TPR decrease (to $47 \%$ vs. to $60 \%, \mathrm{p}<0.05$ ), whereas in the patients no clear CO or TPR pattern was discernible. In follow up, 8 of 10 patients using LBMT reported fewer IOH symptoms. LBMT is a new intervention to attenuate the transient blood pressure decrease after standing up from squatting. IOH patients should be advised about the use of this manoeuvre.
\end{abstract}




\section{INTRODUCTION}

Transient loss of consciousness (TLOC) is a common medical problem, usually caused by intermittent disturbances in neural blood pressure (BP) control [1]. One of its causes is initial orthostatic hypotension $(\mathrm{IOH}) . \mathrm{IOH}$ is defined as symptoms of cerebral and retinal hypoperfusion (e.g. lightheadedness, visual disturbances and/ or syncope) within $15 \mathrm{~s}$ after standing up from a supine, sitting or squatting position caused by an abnormally large transient BP decrease (e.g. $>40 \mathrm{mmHg}$ systolic) [2]. Especially standing up from squatting has been recognised as an acute hemodynamic stressor [3; 4] which can provoke these complaints.

As with vasovagal syncope, for which according to current guidelines history taking rather than tilt testing is of diagnostic importance [5-7], the diagnosis of IOH also depends on a typical history. The active standing test as a diagnostic provocation for IOH has probably an even lower "sensitivity" than the tilt test for vasovagal syncope; the latter is estimated to amount to only $50 \%$ in patients with a classical history $[2 ; 5 ; 7]$.

In a hospital setting IOH is the underlying cause of TLOC in about $3-4 \%$ of syncope cases [2]. Syncope and pre-syncope, regardless of their aetiology may markedly decrease quality of life [8; 9]. Therapeutic management of $\mathrm{IOH}$ is thus an important issue. However the current therapeutic options are limited to general volume measures (e.g. salt loading [10]) that may have hypertensive side effects. The advice to rise slowly [2] may not always be feasible, especially when rising from squatting.

Tensing of leg, buttock and abdominal skeletal muscles, i.e. "lower body muscle tensing" (LBMT), is effective in increasing BP both in patients with postural hypotension due to autonomic failure [11] and during vasovagal reactions [12-14]. LBMT acutely minimises blood pooling in the veins of the lower body and thereby reinfuses blood into the thoracic circulation, enhancing cardiac output (CO) during hypotensive episodes [13; 15]. IOH is thought to be caused by active large skeletal muscle contractions [2]. So although LBMT has been shown effective in other forms of (episodic) hypotension, it is unclear whether this intervention would have any beneficial effects on IOH.

With this information as background, we set out to test the hypothesis that LBMT blunts the BP response to standing up from squatting. In addition we hypothesised that the beneficial effects of LBMT when applied to attenuate $\mathrm{IOH}$ would be caused by an increase in CO. We studied healthy subjects and $\mathrm{IOH}$ patients during standing up manoeuvres from squatting and used a combination of non-invasive continuous BP recording and pulse wave analysis to assess hemodynamic changes. 


\section{METHODS}

We studied 13 patients who were referred to our syncope unit for the evaluation of TLOC (9 males, median height $180 \mathrm{~cm}$ (range 152 - 204), median body weight $77 \mathrm{~kg}$ (range 55 - 97), median age 27 years (range 15 - 59), with a median of 4.5 episodes of syncopes in their lifetimes (range 0 - 100)).

All patients had a clinical diagnosis of IOH based on a consistent history of (pre-)syncope occurring 5 - $15 \mathrm{~s}$ after rising from a supine or squatting position. There was no classic orthostatic hypotension (i.e. $\triangle \mathrm{SBP}>20 \mathrm{mmHg}$ and / or $\triangle \mathrm{DBP}>10 \mathrm{mmHg}, 3$ minutes after standing up) [16]. The median duration of (pre-)syncopal symptoms was 2 years (range 4 months - 10 years). Two patients had experienced frequent pre-syncope after standing up (i.e. daily to weekly) but this had never resulted in loss of consciousness. Of the patients with syncope 4 also had daily pre-syncopal complaints after standing up; 6 experienced such complaints on a weekly basis and one patient had occasional complaints.

\section{Protocol}

BP was measured continuously by finger volume clamp photoplethysmography (Finometer, Finapres Medical Systems, The Netherlands). After $\sim 5 \mathrm{~min}$ free standing the patients squatted for $\sim 1 \mathrm{~min}$, rose within $1 \mathrm{~s}$ and stood for $\sim 1 \mathrm{~min}$. On repeat squat-stand after another $\sim 1 \mathrm{~min}$ of squatting, they performed LBMT for 30 - $60 \mathrm{~s}$ immediately after rising. If patients reported light-headedness or other pre-syncopal symptoms these were documented. LBMT consisted of tensing of all skeletal muscles in the abdomen, buttocks and legs at maximal voluntary capacity for $40 \mathrm{~s}$.

The experiments were performed during the evaluation of the patients for IOH. Patients were positioned in front of the monitor and could observe their BP responses. The duration of squatting varied slightly among patients and was of shorter duration compared to the healthy subjects because of the patient evaluation setting. Repeated squatting for long periods is experienced as uncomfortable by some patients.

After completing the protocol, a subset of 5 patients repeated the squatting protocol twice. (This was done to give them further explanation and instruction.) The first of the three "trials" was used for the comparison described below.

To reduce the potential confounding influence of the fixed order of control and intervention in the patient series, we additionally studied 7 healthy volunteers ( 5 males, median age 27 years (range 25 59), height $179 \mathrm{~cm}$ (range 164 - 202), body weight $74 \mathrm{~kg}$ (range 52 - 85)) who had not experienced significant IOH symptoms over the last year. In a cross-over study design we assessed the effects of LBMT after standing up from squatting. First, all subjects squatted for $2 \mathrm{~min}$, rose within $1 \mathrm{~s}$ and stood 
for 2 min. Standing up from the squatting position was repeated twice. Four randomly assigned subjects performed LBMT after rising from the second squatting, while the remaining 3 subjects performed LBMT after rising from the third squatting.

These experiments were performed in accordance to the standards set in the Declaration of Helsinki after approval by the Medical Ethics Committee of the Academic Medical Center at the University of Amsterdam and obtaining written informed consent.

\section{Analysis}

Off-line, beat-to-beat systolic (SBP) and diastolic (DBP) arterial blood pressure and heart rate (HR) were derived from the arterial pulse wave. Mean arterial pressure (MAP) was the time-integral over the beat-to-beat pressure recording. Corrupted data-points (e.g. artefacts in the continuous BP recording) were identified by visual inspection and omitted $(<2 \%)$. Relative changes in left ventricular stroke volume (SV) were obtained using pulse wave analysis (Modelflow, Finapres Medical Systems, The Netherlands [17]. This method has been validated during active and passive postural stress against thermo-dilution [18], during rapid changes in posture against Doppler ultrasound [19], and during physical counter-manoeuvres against gas re-breathing [15]. CO was HR * $\mathrm{SV}$. Beat-to-beat TPR was MAP $* \mathrm{CO}^{-1}$. After pulse wave analysis all beat-to-beat data were resampled at $1 \mathrm{~Hz}$.

Baseline values were taken from the interval -40 to $-10 \mathrm{~s}$ before each standing up manoeuvre. MAP nadir $\left(\mathrm{MAP}_{\min }\right)$ induced by each manoeuvre was identified and the synchronous $\mathrm{CO}$ and TPR were calculated. All variables are given as median and range. Using Wilcoxon's signed rank test we compared $\mathrm{MAP}_{\min }, \mathrm{CO}$ and TPR respectively, in the patients after standing from squatting without and with LBMT. In the healthy subjects Friedman's repeated measures ANOVA on ranks identified differences between the two squats without and the single squat with LBMT. For all tests a p-value < 0.05 was considered significant.

\section{Follow up}

Using a questionnaire, the perceived effectiveness of LBMT in the patients' daily lives was evaluated addressing the use of the LBMT (daily, weekly/monthly, or never), the frequency of (pre-) syncopal spells after learning LBMT as compared to before (less, same, disappeared), and the perceived benefit from the manoeuvre (some benefit, much benefit; no benefit). 


\section{Results}

After rising from the first squat, the patients' MAP decreased from $110 \mathrm{mmHg}$ (range 88 - 144) to 69 $\mathrm{mmHg}$ (range (53 - 91); SBP from $145 \mathrm{mmHg}$ (range 121 - 201) to $90 \mathrm{mmHg}$ (range 70 - 123); DBP from $88 \mathrm{mmHg}$ (range 69 - 105) to $58 \mathrm{mmHg}$ (range 40 - 76); Figure 1). $\Delta \mathrm{SBP}$ was $>40 \mathrm{mmHg}$ in 12 patients and all experienced pre-syncopal symptoms.

At baseline there were no differences between the first and the second squat in MAP (110 vs. 109 $\mathrm{mmHg}$ ), $\mathrm{CO}$ (6.5 vs. 6.4 arbitrary units) and TPR (1.0 vs. 1.0 arbitrary units). When the stand up was repeated with LBMT, MAP ${ }_{\min }$ was $19 \mathrm{mmHg}$ higher than without LBMT (88 vs. $69 \mathrm{mmHg}, \mathrm{p}<0.05$ ). $\mathrm{SBP}$ at $\mathrm{MAP}_{\min }$ was $111 \mathrm{mmHg}$ (range 67 - 163); DBP $69 \mathrm{mmHg}$ (range 43 - 87)) (Figure 1). $\triangle \mathrm{SBP}$ was $>40 \mathrm{mmHg}$ in 5 patients.

Figure 2 shows the continuous blood pressure recording in a representative patient during consecutive stand up manoeuvres from squatting with and without LBMT.

At group level $(n=13) \mathrm{CO}$ and TPR at $\mathrm{MAP}_{\min }$ did not differ between control and LBMT. In 4 patients the $\mathrm{MAP}_{\min }$ with LBMT was accompanied by a $>10 \%$ higher TPR as compared to without LBMT; and in 8 subjects CO was $>10 \%$ higher. Also in a sub-analysis of patients with a difference of $\mathrm{MAP}_{\min }>10 \mathrm{mmHg}$ between control and LBMT $(\mathrm{n}=5)$ there was no single pattern in TPR and / or $\mathrm{CO}$.

Repeated squat-stand manoeuvres in a subset of 5 subjects revealed a trend for a larger $\triangle \mathrm{MAP}$ after successive squats (Figure 3). However the effect of LBMT seemed to increase over the course of 3 successive trials, suggesting a learning effect (Figure 3).

In the healthy subjects there was also no difference in MAP during the three squats (95 vs. 97 vs. 98 $\mathrm{mmHg}$ ), $\mathrm{CO}$ (5.5 vs. 5.2 vs. 5.2 arbitrary units) or TPR (1.0 vs. 1.1 vs. 1.1 arbitrary units) at baseline. When the subjects used LBMT after standing up, $\mathrm{MAP}_{\min }$ was higher than after either of the two squat-to stand manouevres without intervention $(76 \mathrm{mmHg}$ vs. $63 \mathrm{mmHg}$ and $57 \mathrm{mmHg}, \mathrm{p}<0.05$, Figure 4). This was associated with a higher TPR at $\mathrm{MAP}_{\min }(50 \%$ vs. $45 \%$ and $38 \%$ of baseline, $\mathrm{p}<$ 0.05); $\mathrm{CO}$ at this point did not differ among interventions (Figure 4). Over the interval $20-30 \mathrm{~s}$ after standing up CO was higher during LBMT than without (144\% vs. $100 \%$ of squatted baseline, p < 0.05), TPR did not differ.

In clinical follow-up 12 of 13 patients were contacted after 2 months (range 1-26). Two patients had experienced no IOH symptoms since consulting with us and had not used LBMT. The remaining 10 patients reported using LBMT on a daily to weekly basis both after rising from squatting and from 
supine. In 8 of them the frequency of their complaints had decreased. Nine patients perceived some or much benefit from the manoeuvre in daily life. General comments included that patients sometimes would rise a first time not using LBMT, forcing them to sit or lay down again; on repeat standing up they would use LBMT and experience no symptoms. 


\section{DISCUSSION}

The main new finding of this study is that LBMT attenuated the BP decrease after standing from squatting in both IOH patients and healthy subjects. In the patients, $\mathrm{CO}$ and TPR at MAP $\mathrm{min}_{\text {in }}$ did not differ consistently for LBMT and control. In healthy subjects the underlying mechanism was a blunting of the transient reduction of TPR related to assumption of the upright position. In clinical follow-up patients perceived beneficial effects of LBMT on IOH complaints in daily life. Rising from squatting is an every day orthostatic stress that may result in IOH complaints in otherwise healthy persons [20] and our results are directly applicable to them. We speculate that LBMT will be similarly effective in combating $\mathrm{IOH}$ after standing up from supine.

Testing the initial hemodynamic adaptation standing up from squatting has been used by various groups [21-24]. The manoeuvre's reproducibility has however never been systematically documented. Our findings in both patients (Figure 3) and healthy controls (Figure 4) suggest that the blood pressure decrease after successive standing up manoeuvres from squatting is augmented.

Rossberg \& Peňáz documented a $\triangle \mathrm{MAP}_{\max }$ when standing up after 6 min of squatting of $\sim 45 \mathrm{mmHg}$, [24]. Rickard and Newmann found a $\triangle \mathrm{DBP}$ of $25 \pm 2 \mathrm{mmHg}, 10 \mathrm{~s}$ after standing up from $4 \mathrm{~min}$ of squatting ( $\triangle \mathrm{MAP} 24 \pm 2 \mathrm{mmHg}$ ), but did not document the exact timing of the $\triangle \mathrm{BP}[25]$. During the squat-stand manoeuvre in healthy subjects in our study the maximum $\triangle \mathrm{MAP}(-31 \mathrm{mmHg})$ was somewhat smaller compared to Rossberg \& Peňáz's data which may be related to the shorter squatting period. The nadir of the $\triangle \mathrm{MAP}$ in Rickard \& Newmann's study may have been passed before the measurement. We conclude that based on the heterogeneity of the squat-stand protocols available in the literature, that vary in duration of squatting and standing times $[21 ; 22 ; 24 ; 26]$, there is a need for studies that relate the duration of squatting to its initial (seconds) and prolonged (minutes) hemodynamic effects after standing up.

Several factors may play a role in the immediate decrease in blood pressure when standing up from squatting [2; 22; 24]. Most important is the acute fall in TPR [22], which is caused by a combination of the following factors $[2 ; 24 ; 27] .1)$ The acute decompression of arterial vessels in the legs causes an instantaneous mechanical decrease in vascular resistance. 2) There is an increase of the arteriovenous pressure gradient, due to decompression of the venous vessels. 3) The relative ischemia in the leg muscles during squatting augments the fall in arterial resistance by local factors ("the posttourniquet effect" [22]). 4) The muscle activity during the standing up manoeuvre may promote venous return which can trigger cardiopulmonary pressure receptors and lead to a transient decrease of sympathetic vasoconstrictor outflow [28]. The vastly decreased TPR is not completely offset by the concomitant increase in $\mathrm{CO}[2 ; 22 ; 24]$. 
How LBMT affects the hemodynamic transient in $\mathrm{IOH}$ patients after standing up from squatting is not fully explained by the data from this study, in terms of consistent differences in $\mathrm{CO}$ and/ or TPR between control and intervention. The findings from the healthy subjects however suggest that, unlike our hypothesis that predicted primarily an effect on $\mathrm{CO}$, an increased TPR at $\mathrm{MAP}_{\min }$ may also play a role. In general the mechanical effects of tensing of large muscle groups on TPR (e.g. by "kinking"of arteries) are insignificant because they are off-set by fast (reflex) adaptations of the arteriolar conductance [29]. We suggest that LBMT augments TPR after standing up from squatting because arteriolar conductance is very high at $\mathrm{MAP}_{\min }$ (see above) and unable to further off-set the mechanical effects of LBMT.

After the initial phase, LBMT causes a sustained elevated CO in both patients and healthy subjects. As CO during orthostatic stress is a function of cardiac filling rather than HR [30] this increase of CO is most likely facilitated by an augmented venous return. This finding is also in agreement with previous studies $[15 ; 31]$ and supports the notion that LBMT acts as a natural antigravity suit that optimizes venous return to the heart and thereby optimizes CO. Previous work showed that central command as a determinant of arterial blood pressure [32], does not play a significant role in the effectiveness of physical counter manoeuvres [14].

A limitation to the study design is that we did not use a validated measure to quantify the effect of LBMT on symptoms while measuring BP. However in the IOH patients we found a difference in $\mathrm{MAP}_{\min }$ with LBMT (as compared to control) of $19 \mathrm{mmHg}$ which would generally be accepted as of clinical relevance [33].

Another concern may be that the order in which control and LBMT were performed in the patients was not alternated or randomised. However the results from the series of healthy subjects show that the effect of the LBMT is consistent irrespective of order of control and intervention experiment.

Additional support for the efficacy of LBMT is found in results from the 5 patients who alternated LBMT and control during successive stand up manoeuvres (Figure 3). The results of the follow up (albeit limited) indicate that LBMT may also be effective in daily life.

In conclusion, this study shows that the transient blood pressure decrease after standing up from the squatting position can be attenuated by LBMT. This manoeuvre is an essentially costless, easy to perform intervention without side effects. Future studies may compare LBMT with traditional therapeutic advice for $\mathrm{IOH}$ after rising (e.g. rising slowly) and test its efficacy in daily life. Based on this laboratory study and its limited follow-up data, LBMT seems a worthwhile addition to existing management options. 


\section{Acknowledgements}

We thank the volunteers for participating in this study and Albert R. Oppenhuizen RN and his team of the AMC Outpatient Department for Cardiovascular Medicine for their logistic support and technical assistance. This work is supported by the Netherlands Heart Foundation (C.T.P.K. 2004/T007; WW 99/181) and the Dutch Diabetes Research Foundation (Y.S.K. 2004.00.00). This support is gratefully acknowledged. 


\section{References}

1. Kapoor, W. N. (2000) Syncope. N. Engl. J. Med. 343, 1856-1862

2. Wieling, W., Krediet, C. T., Van Dijk, N., Linzer, M. and Tschakovsky, M. E. (2007) Initial orthostatic hypotension: review on a forgotten condition. Clin. Sci. (Lond). 112, 157-165

3. Barbey, K., Kutscha, W. and Brecht, K. (1966) [Changes of the temporal arterial pulse and the phoniocardiogram in acute orthostatic stress]. Med. Welt 35, 1824-1834

4. O'Donnell, T. V. and McIlroy, M. B. (1962) The circulatory effects of squatting. Am. Heart J. 64, 347-356

5. Sheldon, R., Rose, S., Connolly, S., Ritchie, D., Koshman, M. L. and Frenneaux, M. (2005) Diagnostic criteria for vasovagal syncope based on a quantitative history. Eur. Heart J. 27, 344350

6. Brignole, M., Alboni, P., Benditt, D. G. et al. (2004) Guidelines on management (diagnosis and treatment) of syncope--update 2004. Europace 6, 467-537

7. Colman, N., Nahm, K., Van Dijk, J. G., Reitsma, J. B., Wieling, W. and Kaufmann, H. (2004) Diagnostic value of history taking in reflex syncope. Clin. Auton. Res. 14 Suppl 1, 37-44

8. Linzer, M., Gold, D. T., Pontinen, M., Divine, G. W., Felder, A. and Brooks, W. B. (1994) Recurrent syncope as a chronic disease: preliminary validation of a disease-specific measure of functional impairment. J. Gen. Intern. Med. 9, 181-186

9. Van Dijk, N., Sprangers, M. A., Colman, N., Boer, K. R., Wieling, W. and Linzer, M. (2006) Clinical factors associated with quality of life in patients with transient loss of consciousness. J. Cardiovasc. Electrophysiol. 17, 998-1003

10. Shichiri, M., Tanaka, H., Takaya, R. and Tamai, H. (2002) Efficacy of high sodium intake in a boy with instantaneous orthostatic hypotension. Clin. Auton. Res. 12, 47-50

11. Wieling, W., Van Lieshout, J. J. and Van Leeuwen, A. M. (1993) Physical manoeuvres that reduce postural hypotension in autonomic failure. Clin. Auton. Res. 3, 57-65

12. Kim, K. H., Cho, J. G., Lee, K. O. et al. (2005) Usefulness of physical maneuvers for prevention of vasovagal syncope. Circ. J. 69, 1084-1088

13. Krediet, C. T., De Bruin, I. G., Ganzeboom, K. S., Linzer, M., Van Lieshout, J. J. and Wieling, W. (2005) Leg crossing, muscle tensing, squatting, and the crash position are effective against vasovagal reactions solely through increases in cardiac output. J. Appl. Physiol. 99, 1697-1703

14. Krediet, C. T., Van Dijk, N., Linzer, M., Van Lieshout, J. J. and Wieling, W. (2002) Management of vasovagal syncope: controlling or aborting faints by leg crossing and muscle tensing. Circulation 106, 1684-1689

15. Van Dijk, N., De Bruin, I. G., Gisolf, J. et al. (2005) Hemodynamic effects of leg crossing and skeletal muscle tensing during free standing in patients with vasovagal syncope. J. Appl. Physiol. 98, 584-590

16. Mathias, C. J. (2002) To stand on one's own legs. Clin. Med. 2, 237-245 
17. Jellema, W. T., Imholz, B. P., Van Goudoever, J., Wesseling, K. H. and Van Lieshout, J. J. (1996) Finger arterial versus intrabrachial pressure and continuous cardiac output during headup tilt testing in healthy subjects. Clin. Sci. (Lond). 91, 193-200

18. Harms, M. P., Wesseling, K. H., Pott, F. et al. (1999) Continuous stroke volume monitoring by modelling flow from non-invasive measurement of arterial pressure in humans under orthostatic stress. Clin. Sci. (Lond). 97, 291-301

19. Van Lieshout, J. J., Toska, K., Van Lieshout, E. J., Eriksen, M., Walloe, L. and Wesseling, K. H. (2003) Beat-to-beat noninvasive stroke volume from arterial pressure and Doppler ultrasound. Eur. J. Appl. Physiol. 90, 131-137

20. Krediet, C. T. (2002) Initial orthostatic hypotension in a 37-year old horse rider. Clin. Auton. Res. 12, 404

21. Convertino, V. A., Ratliff, D. A., Crissey, J., Doerr, D. F., Idris, A. H. and Lurie, K. G. (2005) Effects of inspiratory impedance on hemodynamic responses to a squat-stand test in human volunteers: implications for treatment of orthostatic hypotension. Eur. J. Appl. Physiol. 94, 392399

22. Convertino, V. A., Tripp, L. D., Ludwig, D. A., Duff, J. and Chelette, T. L. (1998) Female exposure to high G: chronic adaptations of cardiovascular functions. Aviat. Space Environ. Med. 69, $875-882$

23. Berry, N. M., Rickards, C. A. and Newman, D. G. (2006) Squat-stand test response following 10 consecutive episodes of head-up tilt. Aviat. Space Environ. Med. 77, 1125-1130

24. Rossberg, F. and Penáz, J. (1988) Initial cardiovascular response on change of posture from squatting to standing. Eur. J. Appl. Physiol. 57, 93-97

25. Rickards, C. A. and Newman, D. G. (2003) A comparative assessment of two techniques for investigating initial cardiovascular reflexes under acute orthostatic stress. Eur. J. Appl. Physiol. 90, 449-457

26. Barbey, K. and Barbey, P. (1966) [Blood shift to the lower extremities in acute orthostatic burdening of the circulation]. Med. Welt. 33, 1693-1698

27. Convertino, V. A., Brock, P. J., Keil, L. C., Bernauer, E. M. and Greenleaf, J. E. (1980) Exercise training-induced hypervolemia: role of plasma albumin, renin, and vasopressin. J. Appl. Physiol. 48, 665-669

28. Wieling, W., Harms, M. P., Ten Harkel, A. D., Van Lieshout, J. J. and Sprangers, R. L. (1996) Circulatory response evoked by a $3 \mathrm{~s}$ bout of dynamic leg exercise in humans. J. Physiol. (Lond). 494, 601-611

29. Tschakovsky, M. E. and Sheriff, D. D. (2004) Immediate exercise hyperemia: contributions of the muscle pump vs. rapid vasodilation. J. Appl. Physiol. 97, 739-747

30. Rowell,L.B. (1993) Human cardiovascular control, Oxford University Press, New York

31. Van Lieshout, J. J., Pott, F., Madsen, P. L., Van Goudoever, J. and Secher, N. H. (2001) Muscle tensing during standing: effects on cerebral tissue oxygenation and cerebral artery blood velocity. Stroke 32, 1546-1551 
32. Critchley, H. D., Corfield, D. R., Chandler, M. P., Mathias, C. J. and Dolan, R. J. (2000) Cerebral correlates of autonomic cardiovascular arousal: a functional neuroimaging investigation in humans. J. Physiol. (Lond). 523 Pt 1, 259-270

33. Hainsworth, R. (2004) Pathophysiology of syncope. Clin. Auton. Res. 14 Suppl 1, 18-24 


\section{Figure Legends}

\section{Figure 1}

Hemodyamic changes from baseline, at mean arterial pressure nadir $\left(\mathrm{MAP}_{\min }\right)$ in individual $\mathrm{IOH}$ patients $(n=13)$ in two squat-stand manoeuvres; first without, second with LBMT. Black dots represent median, error bars are $25^{\text {th }}$ and $75^{\text {th }}$ percentile. n.s.: statistically non-significant differences between interventions.

\section{Figure 2}

Continuous blood pressure recording in a 17 year old male $\mathrm{IOH}$ patient during consecutive standing up manoeuvres from squatting. Standing up manoeuvres B, D and F are combined with LBMT. Black bars indicate standing up.

\section{Figure 3}

Continuous systolic and diastolic blood pressures in $5 \mathrm{IOH}$ patients who performed a standing up from squatting 6 times, alternating no intervention (grey) with the LBMT (black). In patient A only during the third trial LBMT seems effective, suggesting a learning effect.

\section{Figure 4}

Hemodyamic changes from baselines, at mean arterial pressure nadir $\left(\mathrm{MAP}_{\min }\right)$ in individual healthy subjects $(n=7)$ in three squatting conditions: two controls (Squat 1, Squat 2), one with LBMT. Black dots represent median, error bars indicate $25^{\text {th }}$ and $75^{\text {th }}$ percentile. ${ }^{*} \mathrm{p}<0.05$, n.s.: statistically nonsignificant differences. 


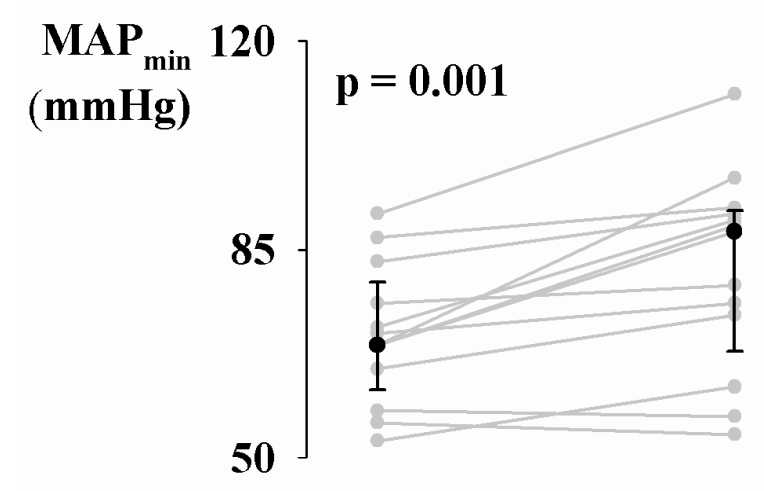

CO (\%) 180 ] n.s.
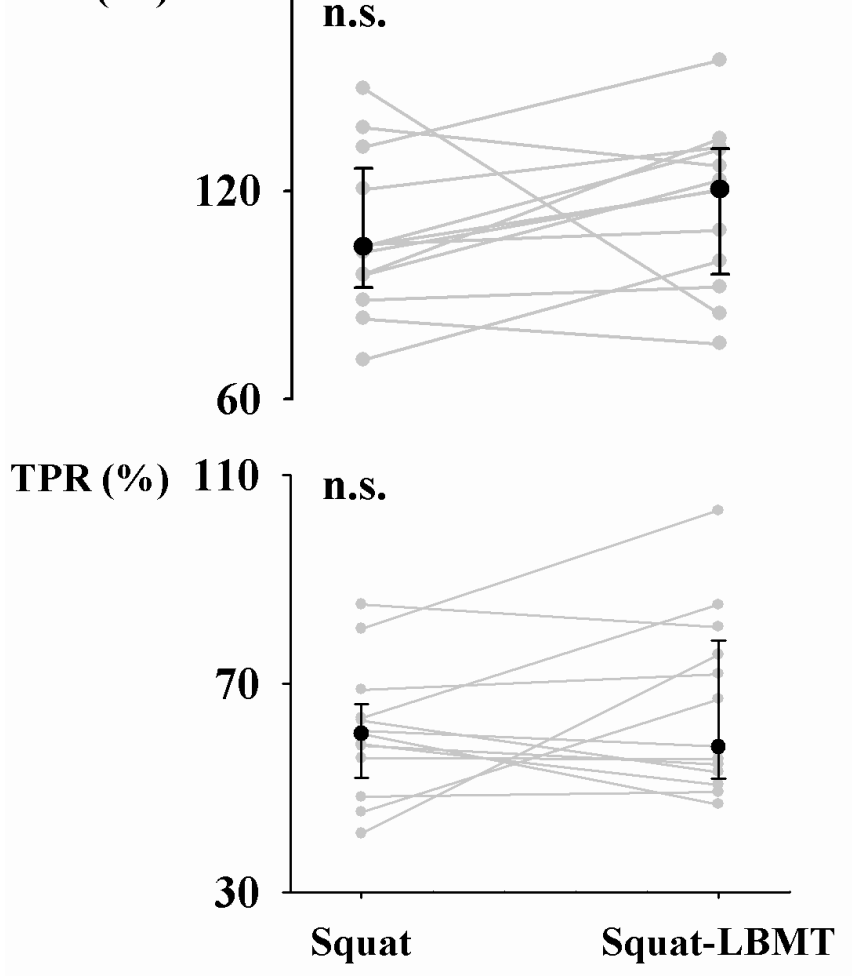

Fig 1 


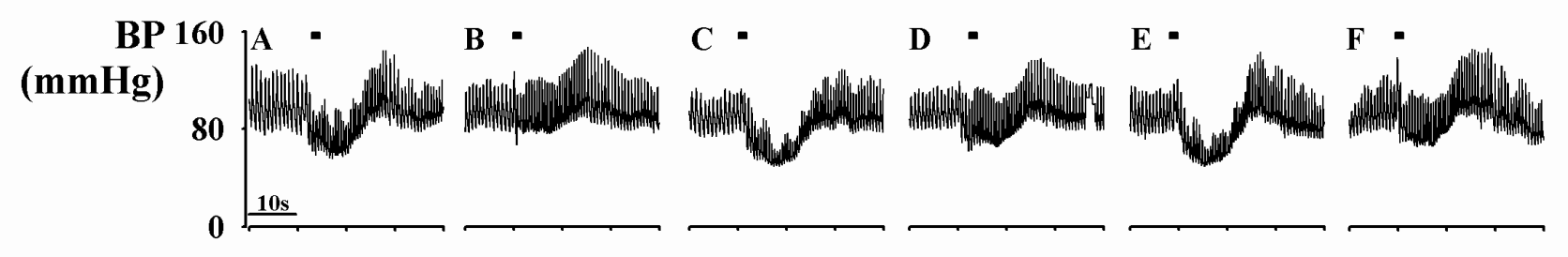

Fig 2 


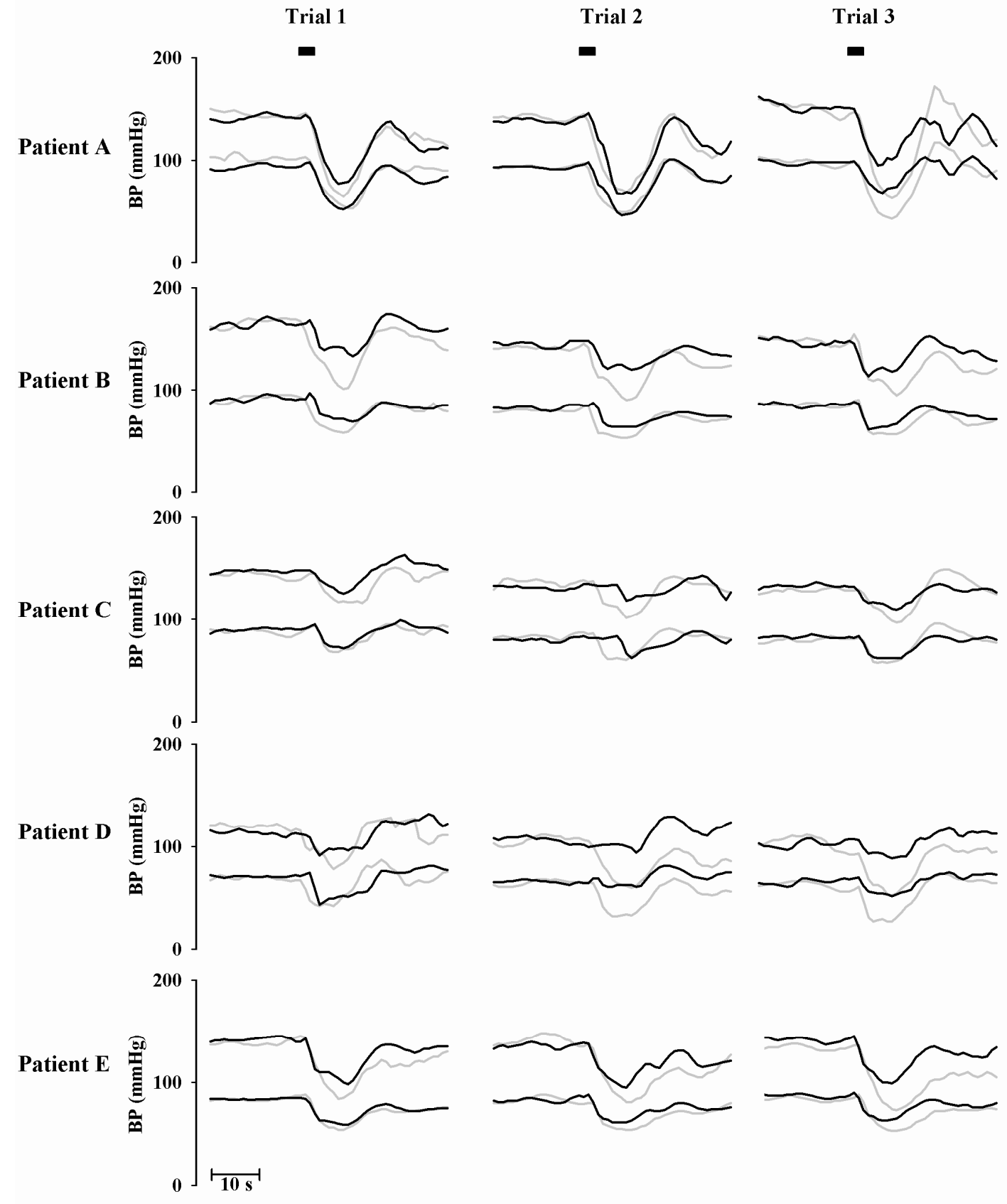

Fig 3 


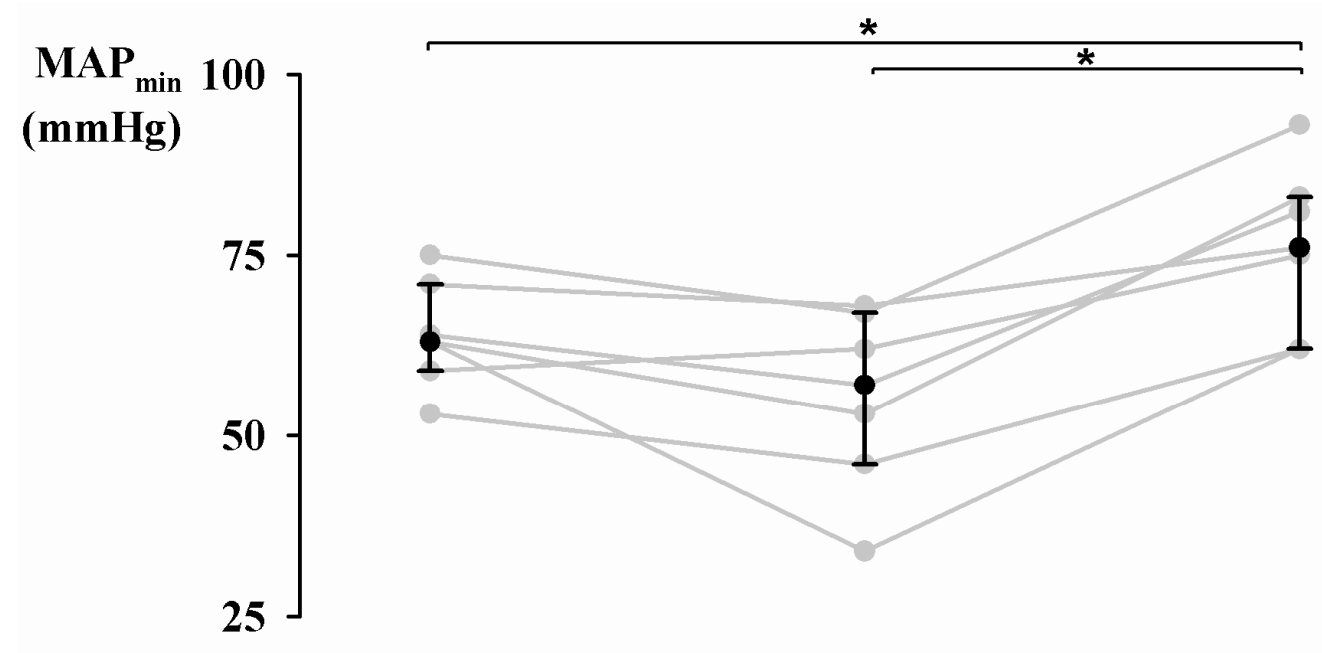

CO (\%) 280$]$ n.s.
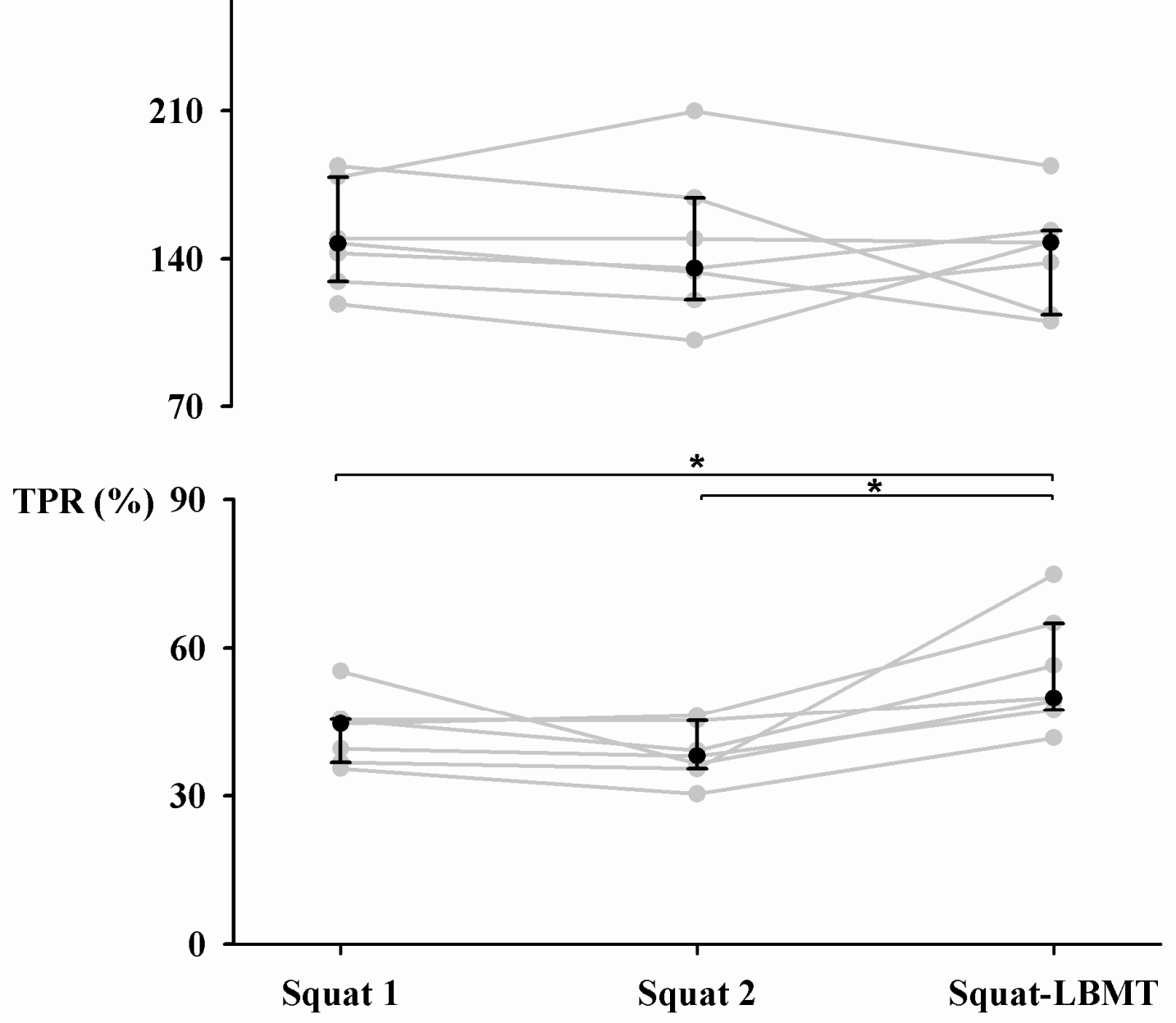

Fig 4 